\title{
Aggregate constrained inventory systems with independent multi- product demand: Control practices and theoretical limitations
}

\author{
Steven De Schrijver ${ }^{\mathrm{a}, \mathrm{b}}$, El-Houssaine Aghezzaf ${ }^{* a}$ and Hendrik Vanmaele ${ }^{\mathrm{a}, \mathrm{b}}$ \\ ${ }^{\mathrm{a}}$ Department of Industrial Management, Faculty of Engineering, Ghent University \\ Technologiepark Zwijnaarde 903, B-9052 Zwijnaarde, Belgium \\ ${ }^{\mathrm{b}}$ MÖBIUS Business Redesign, \\ Kortrijksesteenweg 152, B-9830 Sint-Martens-Latem, Belgium
}

\begin{abstract}
:
In practice, inventory managers are often confronted with a need to consider one or more aggregate constraints. These aggregate constraints result from available workspace, workforce, maximum investment or target service level. We consider independent multi-item inventory problems with aggregate constraints and one of the following characteristics: deterministic leadtime demand, newsvendor, basestock policy, rQ policy and sS policy. We analyze some recent relevant references and investigate the considered versions of the problem, the proposed model formulations and the algorithmic approaches. Finally we highlight the limitations from a practical viewpoint for these models and point out some possible direction for future improvements.
\end{abstract}

Keywords: Multi item, aggregate constraint, inventory system, independent demand

\section{Introduction and motivation}

In practice inventory managers have system wide limitations or goals. Applying a single item approach to attain these goals is not a best practice and neither is it effective to respect system constraints. Sherbrooke (2004) reports that using a system approach on 1.414 spare parts resulted in a $46 \%$ reduction of inventory investment without a decrease in performance. An optimal policy surface can be generated, this is a practical tool to deduct the optimal link between system cost and system service, while respecting the system constraints. Within this article we want to give an overview of the relevant references for the considered policies together with insights in the used algorithms. The usefulness in practice requires the possibility for large data sets and easy implementation, e.g. closed form expressions. The article is organized according the following inventory policies:

- Deterministic leadtime demand.

- Newsvendor: a single period model with a stochastic demand and penalty costs for ordering too much or too little.

- Basestock: an $\mathrm{rQ}$ policy with $\mathrm{Q}=1$, this is relevant when ordering costs are negligible compared with other costs.

- rQ policy: an order of size Q is placed as soon as the inventory position declines to or below the reorder point $r$.

- $\mathrm{sS}$ policy: an order is placed to reach the stock maximum level $\mathrm{S}$ as soon as stock declines to or below reorder point $\mathrm{s}$.

\footnotetext{
* Corresponding author, tel: +329264 55 00, fax: +3292645847 elhoussaine.aghezzaf@ugent.be, steven.deschrijver@mobius.eu,$\underline{\text { hendrik.vanmaele@mobius.eu }}$
} 


\section{Situating the problem}

\subsection{Problem in Scope}

We consider inventory problems with the following formulation:

$$
\begin{array}{ll}
\text { Minimize } & f(\boldsymbol{x})=\sum_{j=1}^{J} f_{j}\left(x_{j}\right) \quad, x_{j} \text { is a vector of m components, } m=1 \text { or } 2 \\
\text { Subject to } \quad g_{n}(\boldsymbol{x})=\sum_{j=1}^{J} g_{n j}\left(x_{j}\right) \leq e_{n} \quad, n=1, \ldots, z \\
x_{j} \in X_{j}=\left[l_{j}, \infty\right] \quad, j=1, \ldots, J, X_{j} \subset \text { of } \mathbb{R}^{m} \text { or } \mathbb{Z}^{m}
\end{array}
$$

The problem described here has $\mathbf{J}$ different items. Each item has $\mathrm{m}$ ( 1 or 2$)$ variables with lower bound $l_{j}$. The variable values are real or in some cases integer. The functions $f_{j}, g_{1 j}, . . g_{z j}$ are functions defined on $R^{m}$ or $Z^{m}$. We will only consider items with independent demand. Each of the problems must have at least one aggregate constraint $(z>=1)$. The items cannot be optimized independently due to the active aggregate constraints.

\subsection{Lagrange multipliers}

Lagrange multipliers can be used to find a solution or as a starting point for an algorithmic approach. The method of Lagrange multipliers can be applied to optimize a function while certain equality constraints need to be respected. A new set of variables $\left(\zeta_{n}\right)$, the Lagrange multipliers, is used in a 'Lagrange function' $Z(x, \zeta)(4)$. We include only active constraints in the Lagrange function. Setting the partial derivatives of the Lagrangian function equal to zero provides a necessary condition for a solution to the constrained problem (5), see Bazaraa, Sherali, Shetty (2006).

$\mathrm{Z}(\boldsymbol{x}, \zeta)=f(\boldsymbol{x})+\sum_{n=1}^{z} \zeta_{n} g_{n}(\boldsymbol{x})$

$\nabla \mathrm{Z}=0$

Everett (1963) points out the usefulness of Lagrange multipliers for optimization in the presence of constraints and the fact that it is not limited to differentiable functions. It is indicated that this method is specifically useful to solve allocation problems with limited resources when faced with independent activities. Patriksson (2008) gives a survey on the continuous nonlinear resource allocation problem, an abundant list of applications is given, amongst which a few inventory cases. The most techniques are based upon iteratively finding the Lagrange multiplier(s). Within each iteration the $x_{j}$ values are calculated or approximated, which allows a check on the constraint validation. Within the algorithms discussed further, creative steps are taken to limit the number of iterations and the complexity to calculate $x_{j}$. Some authors apply other algorithms such as LP and heuristics. In case of integer demand specific enumeration techniques are used or sometimes mixed integer programming, which may work fine for smaller models. Continuous approximations can serve well as lower bound.

\subsection{List of used symbols}

The subscript $j$ refers to item $\mathrm{j}$.

$\begin{array}{ll}c_{j} & \text { Purchase cost } \\ c_{o j} & \text { Overage cost for remaining inventory at period end } \\ c_{u j} & \text { Underage cost for unsatisfied period demand } \\ e_{n} & \text { Upper limit for aggregate constraint } \mathrm{n} \\ f_{j} & \text { Goal function }\end{array}$


$G_{j}{ }_{j} \quad$ Discrete first order loss function of demand distribution

$g_{n j} \quad$ Aggregate constraint function of aggregate constraint $\mathrm{n}$

$h_{j} \quad$ Holding cost, cost to hold one unit in inventory for one unit of time

$J \quad$ Number of items

$j \quad$ Item index, $\mathrm{j}=1, \ldots, \mathrm{J}$

$k_{j} \quad$ Ordering cost, fixed cost to place an order

$l_{j} \quad$ m lower bound value(s) for $\mathrm{x}_{\mathrm{j}}$

$m \quad$ Number of variables per item

$M_{j} \quad$ Renewal function of $\Phi_{j}$

$n \quad$ Aggregate constraint index

$p_{j} \quad$ Shortage cost of dimension (moneys/[quantity-unit- time-unit])

$Q_{j} \quad$ Order Quantity

$r_{j} \quad$ Reorder level for rQ policy

$s_{j} \quad$ Reorder point level for sS policy or target stock level in base stock policy

$S_{j} \quad$ Order up to level of sS policy

$w_{n j} \quad$ Requirement of constrained resource $\mathrm{n}$ by item $\mathrm{j}$

$\boldsymbol{x} \quad$ Matrix with variables for all items

$x_{j} \quad \mathrm{~m}$ variables that determine behavior of item

$z \quad$ Number of aggregate constraints

$\zeta_{n} \quad$ Lagrange variable for constraint $\mathrm{n}$

$Z(x, \zeta) \quad$ The Lagrangian function

$\lambda_{j} \quad$ Mean demand rate

$v_{j} \quad$ Mean of the demand during lead time

$\pi_{j} \quad$ Shortage cost of dimension (moneys/quantity-unit)

$\Phi^{*} \quad(\mathrm{~L}+1)$-fold convolution of $\Phi$ with itself, $\mathrm{L}$ is lead time

$\Phi^{0} \quad$ Standard normal complementary distribution function

$\Phi^{1} \quad$ Standard normal first order loss function

$\Phi^{2} \quad$ Standard normal second order loss function

$\varphi_{j} \quad$ Demand density function

\section{Inventory versions of the problem}

For each of the considered policies we give one model formulation. Afterwards extensions to this basic model are discussed.

\subsection{Deterministic leadtime demand}

Starr, Miller (1962) determine for each item the optimal order quantity to minimize the cost while the average total investment in inventory is limited, see (6), (7) and (8). They also create an 'optimal policy curve' expressing optimal total average inventory for each total number of orders and vice versa. Using Lagrange multipliers a closed form expression is created for this curve.

$z=1, m=1 \rightarrow Q_{j}, l_{j}=1, X_{j} \subset \mathbb{R}$

Minimize $\quad f(x)=\sum_{j=1}^{J}\left[\frac{\lambda_{j} k_{j}}{Q_{j}}+\frac{h_{j} Q_{j}}{2}\right]$

Subject to $\quad g(\boldsymbol{x})=\sum_{j=1}^{J}\left[\frac{c_{j} Q_{j}}{2}\right] \leq e$ 
Zipkin (2000) performs a sensitivity analysis based upon a 'variety index' that embodies the effective variety of the system on this 'inventory-workload trade-off curve'. An increase of this variety index in a large conglomerate is linked to the increasing sales but declining turnover. Hadley, Within (1963) use the Lagrangian principle to handle one or multiple constraints such as average floor space and average number of orders. Page (1976) proposes a grouping procedure, 'The equal order interval method' that outperforms the Lagrangian approach when the maximum investment must be limited. Goyal (1978) improves this heuristic making use of order phasing and a basic replenishment cycle. Rosenblatt (1981) explains that neither of these methods, Lagrange or grouping, really finds the optimal value due to simplifications in both formulations. RosenBlatt, Rothblum (1990) shift towards a penalty like method where extra capacity can be bought. Puerto, Fernandez (1998) construct a multi objective problem using a Pareto-optimal approach. This is a way of doing global sensitivity analysis on the solution space. Haksever, Moussourakis (2005) proposes a mixed integer programming model to deal with multiple linear constraints while making use of piecewise linear approximations. The model chooses between an independent or fixed cycle approach. Test problems with up to 30 items are solved. Boctor (2009) introduces a new mathematical formulation and an efficient heuristic for this inventory replenishment staggering problem. The replenishment cycles must be integer multiples of a basic cycle. Examples with up to 200 items can be solved approximately within seconds while outperforming previous heuristics with $11 \%$ better results.

\subsection{Newsvendor model with a single constraint}

In the work of Hadley, Within (1963) one can end up with negative service levels in case of very tight capacity constraints for the problem given in (9), (10) and (11). Lau, Lau (1996) extend the method so it can handle general demand distributions making use of the Lagrangian method.

$z=1, m=1 \rightarrow Q_{j}, l_{j}=0, X_{j} \subset \mathbb{R}$

Minimize $\quad f(\boldsymbol{x})=\sum_{j=1}^{J}\left(c_{o j} \int_{0}^{Q_{j}}\left(Q_{j}-x\right) \varphi_{j}(x) d x+c_{u j} \int_{Q_{j}}^{\infty}\left(x-Q_{j}\right) \varphi_{j}(x) d x\right)$

Subject to $\quad g(\boldsymbol{x})=\sum_{j=1}^{J} w_{j} Q_{j} \leq e$

In an alternative approach using deterministic optimization by Vairaktarakis (2000) uncertainty is described using interval and discrete demand scenarios. Algorithms are applied for minimax regret objectives to obtain optimal solutions under the defined conditions. AbdelMalek, Montanari (2005a) further analyzes the phenomenon of the lower bounds and divides the solution space in three regions: a non binding constraint region, a binding constraint region where each product can be bought and finally a region with a very strict constraint resulting in zero order sizes for some products. An iterative Lagrangian based method is used whit an approximation of the cumulative distribution. Zhang, Xu, Hua (2009) continue on this work and creates a solution algorithm using a binary search procedure with near optimal solutions for a continuous demand distribution and a good approximate solution for discrete demand. Lower bounds or multiple aggregate constraints is considered as future research. In a 5 item discrete demand test the reached solution has a gap of $2.2 \%$ with the optimal cost, while budget constraint violation is on average 5\%, in case of violation. Abdel-Malek, Montanari, Meneghetti (2008) expand the scope of this problem by integrating a random yield. They refer to this problem as the Gardener Problem: a gardener has a limited acreage 
and must divide this over several possible crops, while demand and yield of the crops is uncertain. An exact solution is reached in case of uniform distribution and an approximate solution in case of other distributions. A 5 item example is demonstrated and validated through simulation.

\subsection{Newsvendor model with multiple constraints}

The primary purpose of Lau, Lau (1996) was to deal with the considerable more difficult multi-constraint problem, see (12), (13) and (14). The primal problem is converted into a dual problem, because typically there is a huge number of items but only a small number of constraints. A solution is constructed using an 'active set of constraints' method that can handle very efficiently systems with a large number of items. The algorithm performance is linked to the number of constraints and the tightness of these constraints. A problem with 1.000 items and 20 constraints is solved within seconds.

$z \geq 2, m=1 \rightarrow Q_{j}, l_{j}=0, X_{j} \subset \mathbb{R}$

Minimize $\quad f(\boldsymbol{x})=\sum_{j=1}^{J}\left(c_{o j} \int_{0}^{Q_{j}}\left(Q_{j}-x\right) \varphi_{j}(x) d x+c_{u j} \int_{Q_{j}}^{\infty}\left(x-Q_{j}\right) \varphi_{j}(x) d x\right)$

Subject to $g_{n}(\boldsymbol{x})=\sum_{j=1}^{J} w_{n j} Q_{j} \leq e_{n}$

$, n=1, \ldots, a$

Abdel-Malek, Montanari (2005b) examine the dual of the solution space with two constraints. An important feature of their approach is its applicability to general probability distribution functions, while it yields an optimum or near optimum solution with a known pre-set error. A 4 item example is solved within 4 iterations with an error on the constraints of less than $0.05 \%$. Abdel-Malek, Areeratchakul (2007) propose a quadratic programming approach, enabling the use of available software packages so that lower bounds and multiple constraints pose no longer an issue. This software can also work with large number of items and offers sensitivity analysis. An example from Lau, Lau (1996) with 7 products and 5 constraints gives nearly the same cost while using familiar software, instead of specific algorithm. Niederhoff (2007) uses separable programming, the simplex method is used to find solutions for nonlinear programs where the objective function and the constraint functions are the sum of functions, where each function involves only one variable. A 10 product example is given, but larger problems form no issue for the software. Zhou, Chen, Wang (2008) introduce a risk factor in this problem defining a $\mathrm{CVaR}$ (conditional value at risk) aggregate constraint that represents a loss function of a portfolio. It is shown that the CVaR model can be represented as a linear program through approximation of the demand density function. A 10 item example is solved and analyzed. Özler, Tan, Karaesmen (2009) use VaR (value at risk) to limit the risk of earning less than a desired target. The VaR constraint is an approximation of the total profit, of different products with independent demand, with a Normal distribution. A non-linear solver is used to solve the case with up to 50 items.

\subsection{Stationary inventory models: base stock models}

Within a spare parts environment it is assumed to have small demand rates and high unit costs, inducing a base stock policy. Sherbrooke (2004) uses a system approach on a set of 1.414 spare parts, what results in a $46 \%$ reduction in inventory investment without a decrease in performance. The problem, see (15), (16) and (17), is solved using marginal analysis. It considers the decrease in backorders by adding one unit, while comparing with the cost of adding one unit for each item. 
$z=1, m=1 \rightarrow s_{j}, l_{j}=-\infty, X_{j}=\mathbb{Z}$

Minimize $\quad f(\boldsymbol{x})=\sum_{j=1}^{J} c_{j} s_{j}$

Subject to $\quad g_{1}(\boldsymbol{x})=\sum_{j=1}^{J} G_{j}^{1}\left(s_{j}\right)$

Thonemann (2002) quantifies the expected improvement in case of a system approach using only a single parameter representation of the unit cost and average demand skewness over all parts. He integrates a time weighted fill rate constraint. Systems with high unit cost skewness profit most from a system approach. Using a 400 item data set it is shown that a high cost skewness, typical in spare parts, gives an improvement between $13 \%$ and $25 \%$. Hill, Pakkala (2007) minimize the cost that includes holding, backorder and order fill rate costs. The order fill rate is the probability that a customer order can be satisfied entirely and immediately from stock, this is relevant in a retail system to prevent extra shipping costs. Through an iterative procedure an approximate solution is reached, a problem with 2.187 items is solved in seconds. Future research to this work can focus on a compound Poisson demand process. Kranenburg, Van Houtum (2007) diversify the target aggregate fill rate over groups of items while commonality exists between groups and a shared stock is used. A heuristic provides a lower bound and an approximate solution. In a case study with 2 groups of 700 items on average $6 \%$ can be saved in spare parts provisioning costs and it takes 13 seconds to run this model.

\subsection{Stationary inventory models: General batch systems (rQ)}

Hadley, Within (1963) touch the problem with aggregate constraints and stochastic demand in case of lot size reorder point models. An iterative procedure is proposed to find the appropriate Lagrange multiplier. The expected number of orders has an upper limit, see (18), (19) and (20). A discussion is held on the difference between a constraint on the average or maximum value of a performance measure. The latter is more complex to solve and is linked to expressing probabilities and risks in constraints.

$z=1, m=2 \rightarrow r_{j} Q_{j}, \quad X_{j} \subset \mathbb{R}$

Minimize

$$
\begin{aligned}
f(\boldsymbol{x}) & =\sum_{j=1}^{J}\left\{\frac{\lambda_{j} k_{j}}{Q_{j}}+h_{j}\left(\frac{Q_{j}}{2}+r_{j}-v_{j}\right)+\left(\frac{h_{j}+p_{j}}{Q_{j}}\right)\left[\Phi^{2}\left(r_{j}\right)-\Phi^{2}\left(r_{j}+Q_{j}\right)\right]\right. \\
& \left.+\frac{\lambda_{j} \pi_{j}}{Q_{j}}\left[\Phi^{1}\left(r_{j}\right)-\Phi^{1}\left(r_{j}+Q_{j}\right)\right]\right\}
\end{aligned}
$$

Subject to $\quad g_{1}(\boldsymbol{x})=\sum_{j=1}^{J} \frac{\lambda_{j}}{Q_{j}} \leq e_{1}$

Ghalebsaz-Jeddi, Shultes, Haj (2004) extend this model and explore the impact of paying purchasing costs when orders arrive. They assume a Normal distribution for the total budget and use an approximate formulation for the expected shortage, which may perform poorly in many situations, see Zipkin (1986). Next they introduce linear and quadratic piecewise approximations. A 2 item example gives approximate results with less iterations than the Hadley, Within (1963) method. Additional constraints are considered as future work. Bera et al. (2009) transform this problem in a multi-objective optimization format using a fuzzy 
chance-constrained technique and surprise function. A minimax distribution free procedure is applied to solve this problem and a 2 item numerical example is also solved. Zhao, Fan, Liu (2007) consider the problem where demand is according a renewal process with customer demands of one unit, e.g. Poisson. The aggregate constraint is the sum of maximum storage for each item separately, as each item will have a fixed location space. An algorithm with polynomial time computation complexity finds the optimal solution and it is tested on an example with 30 items. The extension to aggregate constraints with commonly resources, such as budget, is seen as future research.

\subsection{Stationary inventory models: rQ system without marginal costs}

Marginal cost information for inventory models is not likely available in practice and the most important inventory performance indicators involve system objectives and constraints. Gardner, Dannenbring (1979) minimize the non-service, while respecting the aggregate budget and workforce constraint, see (21), (22), (23) and (24). This is visualized as an optimal policy surface. The formulations for service level and average inventory are approximate formulations. For the problem with one constraint an iterative procedure is used to find the Lagrange variable. In case of two constraints the iterative search for the Lagrange variables is more complex as there interdependency between $r_{j}$ and $Q_{j}$. An application of this technique on a sample of 78.180 items showes that workload can go down $25 \%$ and service increases with $1 \%$ to $6 \%$ without increasing the necessary investment.

$$
\begin{array}{ll}
z \geq 1, m=2 \rightarrow r_{j} Q_{j}, \quad X_{j} \subset \mathbb{R} & \\
\text { Minimize } & f(\boldsymbol{x})=\sum_{j=1}^{J} \frac{\lambda_{j}}{Q_{j}} \int_{r_{j}}^{\infty}\left(x-r_{j}\right) \varphi_{j}(x) d x \\
\text { Subject to } & g_{1}(\boldsymbol{x})=\sum_{j=1}^{J} \frac{Q_{j}}{2}+r_{j}-v_{j} \leq e_{1}, \text { budget constraint } \\
g_{2}(\boldsymbol{x}) & =\sum_{j=1}^{J} \frac{\lambda_{j}}{Q_{j}} \leq e_{2}, \text { workforce constraint }
\end{array}
$$

Schrady, Choe (1971) minimize the long term time weighted backorders while respecting a system budget constraint, although a simplification was made to both formulations. A first solution approach finds the Wilson order sizes and iteratively calculates the reorder levels making use of a Lagrangian. For multiple constraints an exterior penalty function method is proposed, see Bazaraa, Sherali, Shetty (2006). Lenard, Roy (1995) group the different items in families. For each family an aggregate item is chosen for which efficient policy surfaces are drawn, based upon simulation. This approach prevents unacceptable shortage levels for a number of items, which is a possible result in case the aggregate service level is formulated as an arithmetic mean of the individual service levels. Hopp, Spearman, Zhang (1997) aim at minimizing the aggregate inventory investment while respecting a maximum order frequency and a minimum service level in case of batch demand, thus implying integer variables. They present three Lagrangian heuristics approximating inventory performance measures. The two simpler heuristics are closed form expressions, but can perform poorer in cases of low service level and low order frequency. A practical case proves the necessity for lower bounds on the reorder level. An implementation of this heuristic in a 30.000 item system gives a $20 \%$ inventory investment reduction for comparable service levels.

\subsection{Stationary inventory models: General batch systems (sS)}

Mittchel (1988) developes an algorithm based upon an approximation of demand for the periodic review sS problem in a multi-item environment with a service constraint, see (25), 
(26) and (27). The service constraint is not weighted by demand. He indicates that operating costs can be reduced significantly when a uniform service model is no longer used. For a 32 item example with a service level of $85 \%$ cost reductions between $20 \%$ and $39 \%$ are achieved.

$$
\begin{aligned}
& z=1, m=2 \rightarrow s_{j} S_{j}, \quad X_{j} \subset \mathbb{R} \\
& \text { Minimize } \quad f(\boldsymbol{x})=\sum_{j=1}^{J}\left\{h_{j}\left[\int_{0}^{s_{j}-s_{j}} \int_{-\infty}^{s_{j}-y}\left(S_{j}-y-x\right) d \Phi_{j}(x) d M_{j}(y)+\int_{-\infty}^{s_{j}}\left(S_{j}-x\right) d \Phi_{j}^{*}(x)\right]+k_{j}\right\} /\left[1+M_{j}\left(S_{j}-s_{j}\right)\right] \\
& \text { Subject to } \quad g_{1}(\boldsymbol{x})=\sum_{j=1}^{J}\left[\int_{0}^{S_{j}-s_{j}} \Phi_{j}^{*}\left(S_{j}-y\right) d M_{j}(y)+\Phi_{j}^{*}\left(S_{j}\right)\right] /\left[1+M_{j}\left(S_{j}-s_{j}\right)\right] \leq e_{1}
\end{aligned}
$$

Scheider, Rinks (1989) uses asymptotic properties from the renewal theory to approximate the optimal solution. Besides the service constraint, two other aggregate constraints are added: one on maximum workload and another on maximum storage room. An iterative grid search is performed for the Lagrange multiplier values. For an 100 item system the results are visualized in an optimal policy surface chart that shows the tradeoff between cost and service level, while respecting the workload and storage constraint. Cohen et al. (1992) add shortage costs and use a demand weighted fill rate constraint. There is no backlogging, so unmet demand is lost. A greedy algorithm is created to find near-optimal solutions for the approximate problem formulation. It is tested on a 4 item part and showes a small error of $3 \%$ in case of low service levels, but larger errors up to $17 \%$ in case of high service levels compared to a lower bound value.

\section{Concluding remarks}

Within a broad range of inventory policies we see a practical need for a system approach, rather than an item approach. This enables the manager to realize his goals with an optimal mix between cost and service while confronted with limited resources such as workspace, workforce or investment. For managers this can be expressed in optimal system policy curves. Test cases with a significant number of items report cost decreases from $10 \%$ up to $46 \%$. Approximations, in performance measures and leadtime demand, are not always without risk. Lagrangian multipliers are often used as an initial approach, as it is attractive because a line search enables the user to solve this separable problem, in case of a single constraint. For the policies where one variable describes the behavior of one item we see that a step is made towards linear or quadratic programming. This give the practical user the ability to model large real life problems, do sensitivity analysis and include multiple constraints if necessary. In case of discrete demand complexity is higher and lower bounds and heuristics are used. Future work might focus on exact formulation of performance measures and demand distributions. Within discrete demand we see referrals to compound Poisson demand as a next step and also the use of multiple constraints.

\section{References}

1. Abdel-Malek, L.L., Areeratchakul, N., 2007. A quadratic programming approach to the multi-product newsvendor problem with side constraint. European Journal of Operational Research, 176(3), 1607-1619.

2. Abdel-Malek, L.L., Montanari, R., 2005a. An analysis of the multi-product newsboy problem with a budget constraint. International Journal of Production Economics, 97(3), 296-307. 
3. Abdel-Malek, L.L., Montanari, R., 2005b. On the multi-product newsboy problem with two constraints. Computers \& Operations Research, 32(8), 2095-2116.

4. Abdel-Malek, L.L., Montanari, R., Meneghetti, D., 2008. The capacitated newsboy problem with random yield: The Gardener Problem. International Journal of Production Economics, 115(1), 113-127.

5. Bazaraa, M.S., Sherali, H.D., Shetty, C.M., 2006. Nonlinear programming, Theory and algorithms, Third edition. John Wiley \& Sons, Hoboken, New Jersey.

6. Bera, U.K., Rong, M., Mahapatra, N.K., Maiti, M., 2009. A multi-item mixture inventory model involving random lead time and demand with budget constraint and surprise function. Applied Mathematical Modelling, 33(12), 4337-4344.

7. Boctor, F.F., 2009. Offsetting inventory replenishment cycles to minimize storage space. European Journal of Operational Reserach, 203(2), 321-325.

8. Cohen, M.A., Kleindorfer, P.R., Lee, H.L., Pyke, D.F., 1992. Multi-item service constrained (s, S) policies for spare parts logistics systems. Naval Research Logistics, 39(4), 561-577.

9. Everett, H., 1963. Generalized Lagrange Multiplier Method for Solving Problems of Optimum Allocation of Resources. Operations Research, 11(3), 399-417.

10. Gardner, E., Dannenbring, D., 1979. Using optimal policy surfaces to analyze aggregate inventory tradeoffs. Management Science, 25(8), 709-720.

11. Ghalebsaz-Jeddi, B., Shultes, B.C., Haj, R., 2004. A multi-product continuous review inventory system with stochastic demand, backorders, and a budget constraint. European Journal of Operational Research, 158(2), 456-469.

12. Goyal, S.K., 1978. A Note on Multi-Product Inventory Situations with One Restriction. The Journal of the Operational Research Society, 29(3), 269-271.

13. Hadley, G., Within, T.M., 1963. Analysis of Inventory Systems. Prentice Hall, New Jersey.

14. Haksever, C., Moussourakis, J., 2005. A model for optimizing multi-product inventory systems with multiple constraints. International Journal of Production Economics, 97(1), 18-30.

15. Hill, R.M., Pakkala, T.P.M., 2007. Base stock inventory policies for a multi-item demand process. International Journal of Production Economics, 109(1-2), 137-148.

16. Hopp, W.J., Spearman, M.L., Zhang, R.Q., 1997. Easily implementable inventory control policies. Operations Research, 45(3), 327-340.

17. Kranenburg, A.A., Van Houtum, G.J., 2007. Effect of commonality on spare parts provisioning costs for capital goods. International Journal of Production Economics, 108(1-2), 221-227.

18. Lau, H.S., Lau, A.H., 1996. The news stand problem: A capacitated multiple-product single-period inventory problem. European Journal of Operational Research, 94(1), $29-42$.

19. Lenard, J.D., Roy, B., 1995. Multi-item inventory control: A multicriteria view. European Journal of Operational Research, 87(3), 685-692.

20. Mittchel, J.C., 1988. Multi-item inventory systems with a service objective. Operations Research, 36(5), 747-755.

21. Niederhoff, J.A., 2007. Using separable programming to solve the multi-product multiple ex-ante constraint newsvendor problem and extensions. European Journal of Operational Research, 176(2), 941-955.

22. Özler, A., Tan, B., Karaesmen, F., 2009. Multi-product newsvendor problem with value-at-risk considerations. International Journal of Production Economics, 117(2), 244-255. 
23. Page, E., 1976. Multi-Product Inventory Situation with One Restriction. Operations Research Quarterly, 27(4), 815-834.

24. Patriksson, M., 2008. A survey on the continuous nonlinear resource allocation problem. European Journal of Operational Research, 185(1), 1-46.

25. Puerto, J., Fernandez, F.R., 1998. Pareto-optimality in classical inventory problems. Naval Research Logistics, 45(1), 83-98.

26. Rosenblatt, M.J., 1981. Multi-item inventory system with budgetary constraint: a comparison between the Lagrangian and the fixed cycle approach. International Journal of Production Research, 19(4), 331-339.

27. RosenBlatt, M.J., Rothblum, U.G., 1990. On the Single Resource Capacity Problem for Multi-Item Inventory Systems. Operations Research, 38(4), 686-693.

28. Scheider, H., Rinks, D.B., 1989. Optimal policy surfaces for a multi-item inventory problem. European Journal of Operational Research, 39(2), 180-191.

29. Schrady, D.A., Choe, U.C., 1971. Models for multi-item continuous review inventory policies subject to constraints. Naval Research Logistics Quarterly, 18(4), 451-463.

30. Sherbrooke, C.C., 2004. Optimal inventory modeling of systems, second edition. Dordrecht, The Netherlands, Kluwer Academic Publishers Group.

31. Starr, M., Miller, D., 1962. Inventory Control: Theory and Practice. Prentice-Hall, Englewood Cliffs, NJ.

32. Thonemann, U.W., 2002. Easy Quantification of Improved Spare Parts Inventory Policies. Management Science, 48(9), 1213-1225.

33. Vairaktarakis, G.L., 2000. Robust multi-item newsboy models with a budget constraint. Internation Journal of Production Economics, 66(3), 213-226.

34. Zhang, B., Xu, X., Hua, Z., 2009. A binary solution method for the multi-product newsboy problem with budget constraint. International Journal of Production Economics, 117(1), 136-141.

35. Zhao, X., Fan, F., Liu, X., 2007. Storage-space capacitated inventory system with (r, Q) policies. Operations Research, 55(5), 854-865.

36. Zhou, Y., Chen, X., Wang, Z., 2008. Optimal ordering quantities for multi-products with stochastic demand: Return-CVaR model. International Journal of Production Economics, 112(2), 782-795.

37. Zipkin, P.H., 1986. Inventory Service-Level Measures: Convexity and Approximation. Management Science, 32(8), 975-981.

38. Zipkin, P.H., 2000. Foundations of Inventory Management. McGraw-Hill, Signapore. 